\title{
Magnetic resonance imaging features in twin neonates with nonketotic hyperglycinemia
}

\author{
Serhat Guler ${ }^{1}$, Alpay Alkan², Serpil Kurtcan², Sinem Aydın²
}

\author{
${ }^{1}$ Department of Pediatric Neurology, Faculty of Medicine, Bezmialem Vakif University, \\ Istanbul, Turkey \\ 2Department of Radiology, Faculty of Medicine, Bezmialem Vakif University, Istanbul, \\ Turkey
}

Submitted: 12 April 2016

Accepted: 12 May 2016

Arch Med Sci Civil Dis 2016; 1: e43-e44

DOI: 10.5114/amscd.2016.60160

Copyright $\odot 2016$ Termedia \& Banach

Nonketotic hyperglycinemia (NKH) is an autosomal recessive inborn error of metabolism caused by a defect in the glycine cleavage system [1]. Magnetic resonance imaging (MRI) findings of neonatal nonketotic hyperglycinemia have rarely been described in the literature, with only a few individual cases or small series reported. In this article, we present twin cases of neonatal onset nonketotic hyperglycinemia, imaged at 3 days of age, and discuss characteristic MRI findings. To our knowledge, this is the only report of such complete MRI analysis in twin neonates.

The female monochorionic diamniotic twin patients were born at 34 weeks gestation by spontaneous vaginal delivery. The pregnancy was uncomplicated. Their parents were nonconsanguineous, with no family history of metabolic or neurologic disease. The Apgar score for the first patient was $7 / 9$ and for the second patient was 6/9. The birth weight of the first patient was $2400 \mathrm{~g}$ and that of the second patient was $2150 \mathrm{~g}$. At 2 days old, they were transferred to our Neonatal Intensive Care Unit for hypotonia, cyanosis, poor feeding, and myoclonic seizures. They were hypotonic and lethargic and had unresponsive neonatal reflexes. The first patient had persistent hiccups and myoclonic convulsions. The second patient had myoclonic convulsions and respiratory depression.

The diagnosis of NKH was made based on a markedly elevated CSF/ plasma glycine ratio $(0.24,0.19$ respectively) at age 7 days. The normal ratio is less than 0.08 [2]. Brain MRI was performed at 3 days of age in our patients. Both patients had progressive cortical and white matter atrophy. The corpus callosum was thin and volume loss was both supraand infratentorial. In T2-weighted images the appearance of the white matter myelination was inappropriate for age. In the first patient, there were subependymal T1-hyperintense and T2-hypointense areas and ventriculomegaly congruent with germinal matrix grade 3 hemorrhage. Hypoplasia of the corpus callosum, cystic dilatation of the fourth ventricle that may hemorrhage and cerebellar volume loss were also present (Figure 1). The second patient's MRI also showed lack of normal myelination for age, hypoplasia of the corpus callosum, ventriculomegaly, retrocerebellar cystic dilatation and cerebellar volume loss (Figure 2). However, intracranial hemorrhage was not observed.

Structural cerebral abnormalities observed in NKH include cerebral atrophy, callosal dysgenesis, posterior fossa cysts and ventriculomegaly $[3,4]$. The improvement of hydrocephalus in patients with NKH has been in particular associated with the existence of large retrocerebellar cysts

\author{
Corresponding author: \\ Serhat Guler \\ Department \\ of Pediatric Neurology \\ Faculty of Medicine \\ Bezmialem Vakif University \\ Adnan Menderes blv \\ Fatih, Istanbul, Turkey \\ Phone: +90 2124531700-7970, \\ +90 5057588993 \\ E-mail: serhatguler@ \\ hotmail.com
}



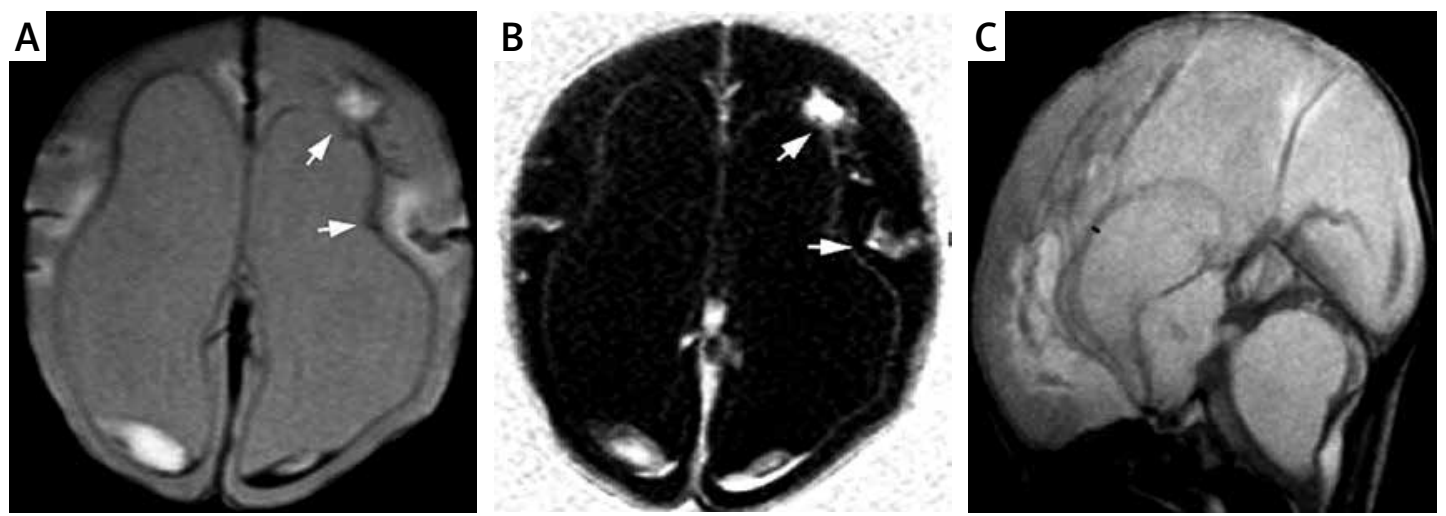

Figure 1. In the first patient, axial T2-weighted (A) and T1-weighted (B) images show progressive cortical and white matter atrophy. Subependymal T1-hyperintense and T2-hypointense areas (arrow) and ventriculomegaly congruent with germinal matrix grade 3 hemorrhage are observed. In the sagittal T2-weighted images (C) the hypoplasia of the corpus callosum and cystic dilatation of the fourth ventricular are clearly visible
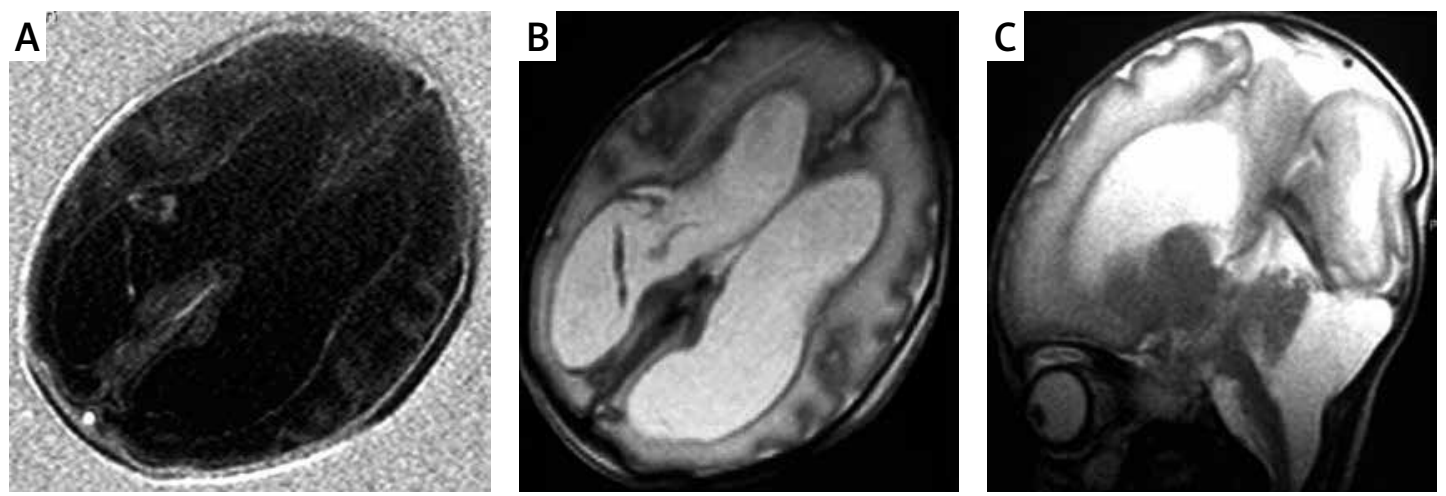

Figure 2. In the second patient, axial T1-weighted (A) and T2-weighted (B) images also showed lack of normal myelination for age, ventriculomegaly, hypoplasia of the corpus callosum, and retrocerebellar cystic dilatation (C)

$[4,5]$. On the third day of life, cranial MRI of the twins showed callosal hypoplasia and ventriculomegaly. Germinal matrix hemorrhage was observed in just one of the twins. In the literature, the relation between germinal matrix hemorrhage and $\mathrm{NKH}$ has not been reported yet. Because of that, the germinal matrix hemorrhage was not associated with either prematurity or $\mathrm{NKH}$.

Brain MRI shows clear pathological changes in children with NKH. The MRI data of 3-day-old twin neonates with NKH show structural cerebral abnormalities. An early diagnosis, though it does not change the disease course, allows proper genetic counseling, with the possibility of prenatal diagnosis. Another benefit of diagnosis is that having a definite diagnosis with this phenotype prevents further excessive investigations for other genetic or metabolic etiologies.

\section{Conflict of interest}

The authors declare no conflict of interest.

\section{References}

1. Manley BJ, Sokol J, Cheong JL. Intracerebral blood and MRS in neonatal nonketotic hyperglycinemia. Pediatr Neurol 2010; 42: 219-22.

2. Hennermann JB. Clinical variability in glycine encephalopathy. Future Neurol 2006; 1: 621-30.

3. Dobyns WB. Agenesis of the corpus callosum and gyral malformations are frequent manifestations of non-ketotic hyperglycinemia. Neurology 1989; 39: 817-20.

4. Van Hove JL, Kishnani PS, Demaerel P, et al. Acute hydrocephalus in nonketotic hyperglycemia. Neurology 2000; 54: 754-6.

5. Yis U, Kurul S, Dirik E. Non-ketotic hyperglycinemia and acquired hydrocephalus. Pediatr Neurol 2009; 40: 138-40. 